\title{
Auger Microanalysis in Spacecraft Tribology
}

\author{
J. R. Lince
}

Micro/Nano Technology Department, Space Materials Laboratory, The Aerospace Corporation, PO Box 92957, Los Angeles, CA 90009-2957

Lubricants and antiwear coatings are used in every aspect of our lives where moving parts are involved, from automobiles to computer disk drives. In spacecraft, tribological materials are especially critical because they cannot be replenished once launched into orbit, and are used on precision devices that must operate effectively for up to twenty years. Typical spacecraft moving mechanical assemblies (MMAs) are attitude control devices (e.g., gyroscopes), solar array deployment mechanisms, and gimbals for precision pointing of antennas and sensors.

Chemical degradation of tribological materials is one of the most important failure mechanisms of spacecraft MMAs. Tribochemical interactions generally occur in a thin layer between contacting surfaces, often less than a nanometer in thickness. Critical to studying these interactions is Auger Microanalysis. It involves scanning an electron beam on a surface, similar to a scanning electron microscope (SEM). However, instead of analyzing emitted $\mathrm{X}$ rays using energy dispersive spectroscopy (EDS) - commonly used with SEMs - low energy Auger electrons (generally in the range 50 to $2000 \mathrm{eV}$ ) are collected. These low energy electrons originate in the topmost 0.5 to $2 \mathrm{~nm}$ of the surface region, ideal for the analysis of tribological contacts. In addition, Auger Microanalysis can exhibit better lateral resolution than EDS because the electron interaction volume decreases with decreasing depth into the sample.

Auger Microanalysis has proven especially useful in studying the chemical interaction of lubricant additives with lubricated surfaces. For example, bearings were tested with the common spacecraft lubricant Pennzane oil formulated with the antiwear additive lead naphthenate (Pbnp). Lubricated steel bearings were used as is, or coated with a hard lubricious diamond-like carbon (DLC) coating [1]. Fig. 1a shows Auger electron spectra obtained from an uncoated steel bearing after testing. Significant amounts of $\mathrm{Pb}$ seen in the wear track indicate that Pbnp has chemisorbed onto the bearing surface. In contrast, much smaller amounts of $\mathrm{Pb}$ are seen on the surface of a highly hydrogenated DLC-coated bearing (see Fig. 1b). This indicates a much smaller chemical interaction (i.e., physisorption), because similar amounts of $\mathrm{Pb}$ are seen on lubricated but untested surfaces.

Thin solid lubricant coatings based on sputter-deposited molybdenum disulfide $\left(\mathrm{MoS}_{2}\right)$ are widely used on spacecraft. Auger Microanalysis was used to understand the lubrication mechanism of cosputtered $\mathrm{Au} / \mathrm{MoS}_{2}$ [2]. Fig. 2a shows a secondary electron image of a tested steel coupon coated with $\mathrm{Au} / \mathrm{MoS}_{2}$, while Figs. 2b and 2c show Auger maps for Au NVV and S LMM Auger transitions. The Auger maps show that there is a large reduction in $\mathrm{Au}$ and an increase in $\mathrm{MoS}_{2}$ at the surface in the contact region. This result, along with additional Auger studies, showed that lubrication is provided by a pure, one-nanometer thick $\mathrm{MoS}_{2}$ film in the contact region.

References

[1] J. R. Lince et al., Journal of ASTM International 5(1) (January 2008), Paper No. JAI101223.

[2] J. R. Lince, Tribol. Lett., 17(3) (2004) 419. 
a

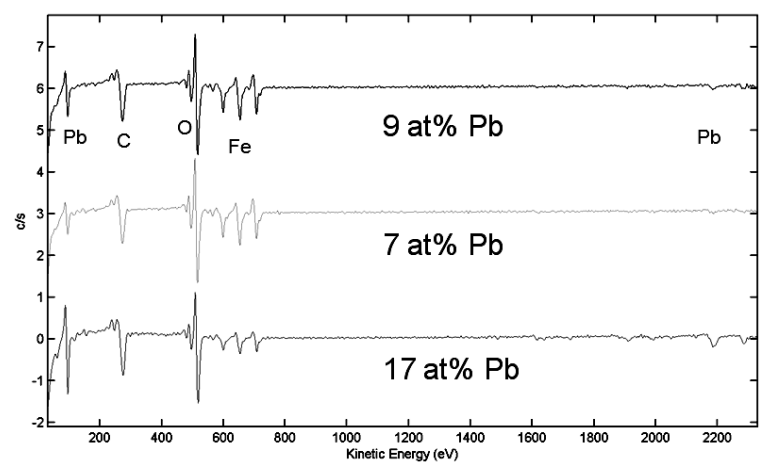

b

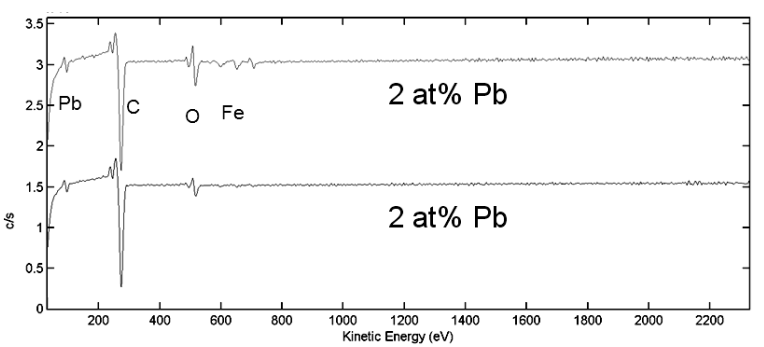

FIG. 1. Auger electron spectra obtained inside the wear tracks of steel thrust bearings. Shown are spectra for (a) an uncoated bearing, and (b) a bearing coated with highly hydrogenated DLC. The $\mathrm{Pb}$ comes from the lead naphthenate in the oil, a common antiwear lubricant additive for spacecraft.

$\mathbf{a}$

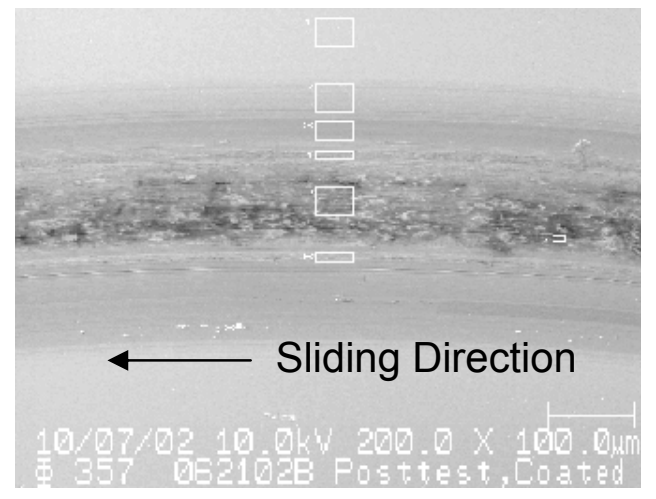

b

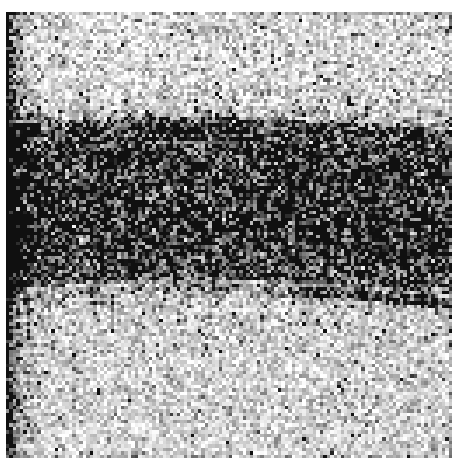

c

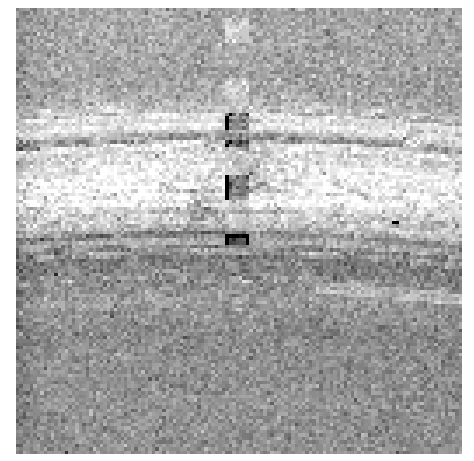

FIG. 2. The wear track after tribological testing of a sputter-deposited $\mathrm{Au} / \mathrm{MoS}_{2}$ coating. Shown is (a) a secondary electron image of the wear track, along with (b) Au and (c) S Auger Maps in the same region. The maps indicate the presence of a surface film that consists of pure $\mathrm{MoS}_{2}$ in the contact region.

This work was supported under The Aerospace Corporation's Mission Oriented Investigation and Experimentation program, funded by the U.S. Air Force Space and Missile Systems Center under Contract No. FA8802-09-C-0001.

(C) The Aerospace Corporation 2010 